\title{
Triplet Shelving in Fluorescein and its Derivatives Provides Delayed, Background-Free Fluorescence Detection
}

\author{
Aida A. Demissie, and Robert M. Dickson*
}

School of Chemistry \& Biochemistry and Petit Institute of Bioengineering and Bioscience,

Georgia Institute of Technology, Atlanta, GA 30332-0400

*Email: dickson@chemistry.gatech.edu

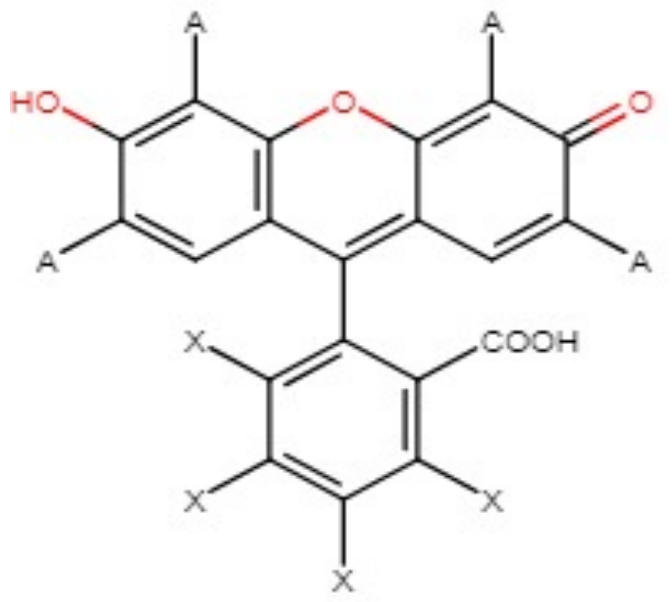

\begin{tabular}{|l|l|}
\hline Fluorescein & $\mathrm{A}=\mathrm{H}$ \\
& $\mathrm{X}=\mathrm{H}$ \\
\hline Rose bengal & $\mathrm{A}=\mathrm{I}$ \\
& $\mathrm{X}=\mathrm{Cl}$ \\
\hline Erythrosin $\mathrm{B}$ & $\mathrm{A}=\mathrm{I}$ \\
& $\mathrm{X}=\mathrm{H}$ \\
\hline Eosin $\mathrm{Y}$ & $\mathrm{A}=\mathrm{Br}$ \\
& $\mathrm{X}=\mathrm{H}$ \\
\hline
\end{tabular}

Figure S1: Structure of xanthenes and the corresponding halogen substituents for each. 


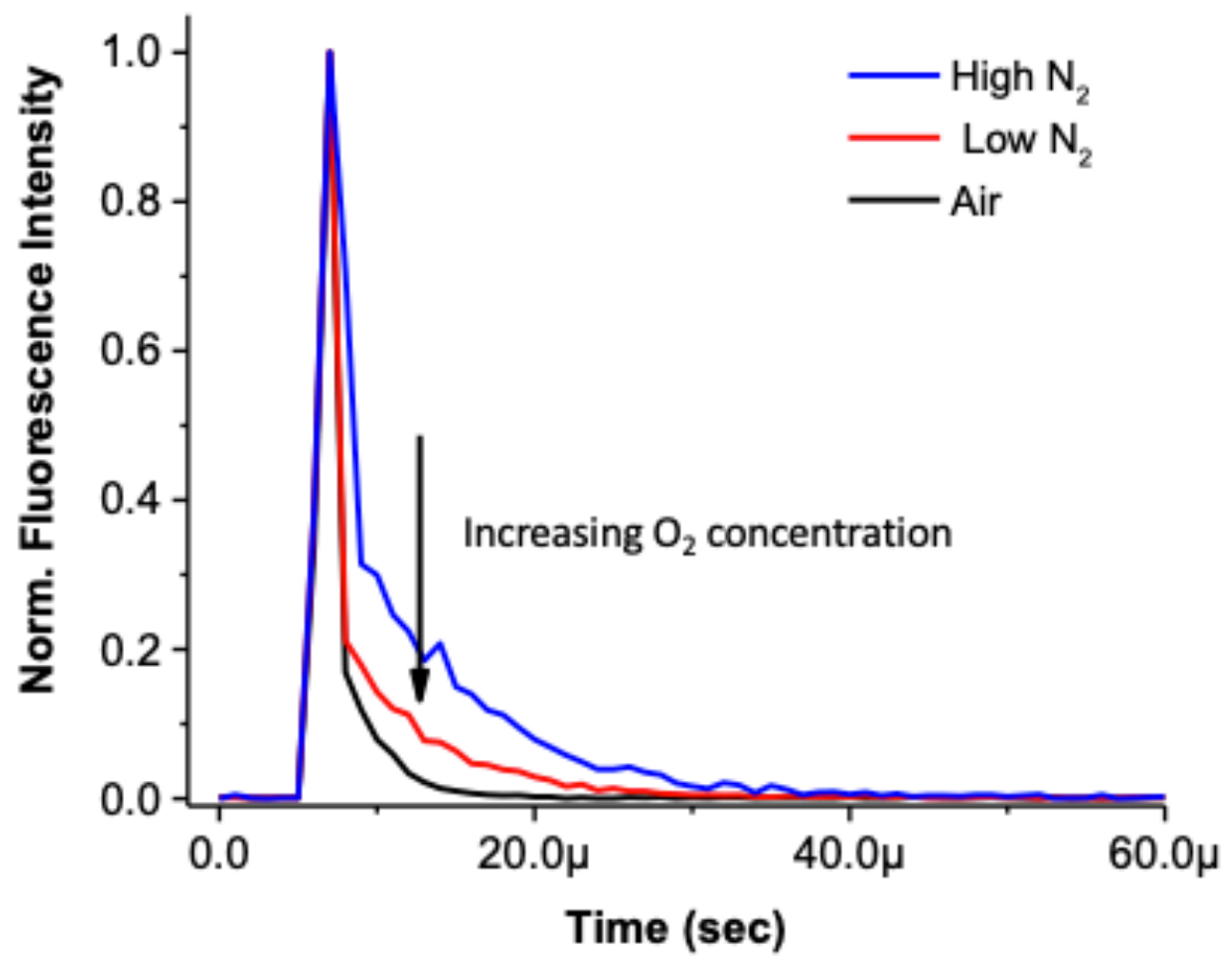

Figure S2: Fluorescence histogram of rose bengal solution under pulsed primary $(10 \mathrm{kHz})$ and CW secondary coillumination under varying levels of oxygen. 
Table S1. Photophysical parameters used for simulation

\begin{tabular}{|l|l|}
\hline Fluorescence lifetime $^{*}\left(k_{f}^{-1}\right)$ & $1.7 \mathrm{~ns}(\mathrm{PVA})$ \\
\hline Fluorescence quantum yield $\left(\Phi_{\mathrm{F}}\right)$ & $\mathrm{RB}=0.05 ; \mathrm{EB}=0.1 ; \mathrm{EY}=0.3 ; \mathrm{Flu}=0.9$ \\
\hline Triplet quantum yield $^{1-2}\left(\Phi_{\mathrm{T}}\right)$ & $\mathrm{RB}=0.93 ; \mathrm{EB}=0.85 ; \mathrm{EY}=0.45 ; \mathrm{Flu}=0.02$ \\
\hline Reverse intersystem crossing quantum yield & \\
\hline
\end{tabular}

\section{Fluorescein dark state lifetime determination:}

Upon absorption of primary laser there is a rapid decrease in fluorescence intensity as molecules transition into the triplet state. The lifetime of the observed fluorescence decay is a function of the rates into and out of the triplet state and depends linearly on the primary laser intensity (no secondary laser used). The rate into the dark state is a function of the primary excitation intensity ( $488 \mathrm{~nm}$ for fluorescein) while the rate out of the dark state is a sum of the secondary induced depopulation of the dark state and the natural decay rate out of the dark state. If no secondary laser is used, however, the rate out the dark state is equal to the natural decay rate given by $k_{o f f}^{0}$. In this scenario, the fluorescence decay observed is described by the below equation; where $\mathrm{I}_{\text {pri }}$ and $\sigma_{\text {isc }}$ are the primary laser intensity and the action cross-section, respectively.

$$
k_{\text {measured }}=\frac{\sigma_{I S C} I_{P r i}}{h v}+k_{o f f}^{0}
$$

Plotting the measured decay vs the primary intensity used will give the natural decay rate $k_{o f f}^{0}$, as the y-intercept. Using this method, the measured rate was found to fit to a bi-exponential give timescales of $12 \mathrm{~ms}$ and $44 \mathrm{~ms}$. Considering the high photobleaching observed in PVA 
immobilized fluorescein, the slower time scale was taken as representing the photobleaching rate.

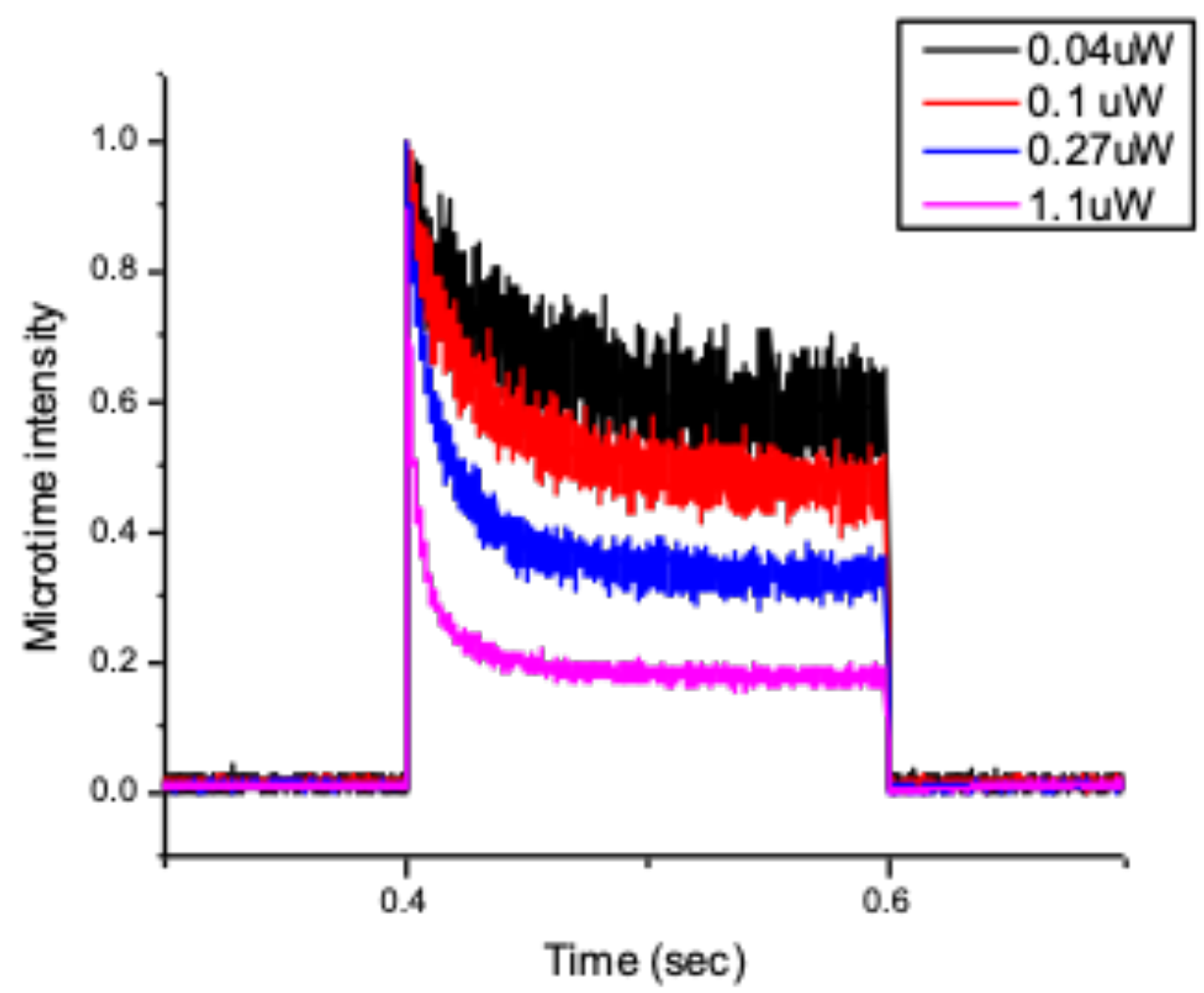

Figure S3: Fluorescence of fluorescein under square wave-modulated $488 \mathrm{~nm}$ primary excitation at varying intensities.

\section{Phosphorescence lifetime determination:}

Phosphorescence for each dye was measured by using simultaneous fluorescence lifetime/phosphorescence lifetime measurement technique. In brief, 532nm (100ps, 10MHz) excitation pulses were modulated at $50 / 100 \mathrm{~Hz}$, and fluorescence/phosphorescence data was collected using a long pass filter. During the on periods of the modulation cycle fluorescence can be measured while phosphorescence measurements are done during the off periods (after fluorescence has decayed) so as to avoid fluorescence noise. Moreover, using multiple excitation pulses allows for sufficient buildup of triplet state giving much stronger phosphorescence. 
Because of the fast nature of the fluorescence, no fluorescence is collected in the microseconds to millisecond window between excitation pulses during which phosphorescence is collected.

Figure S4 shows the phosphorescence histogram of rose bengal in PVA.
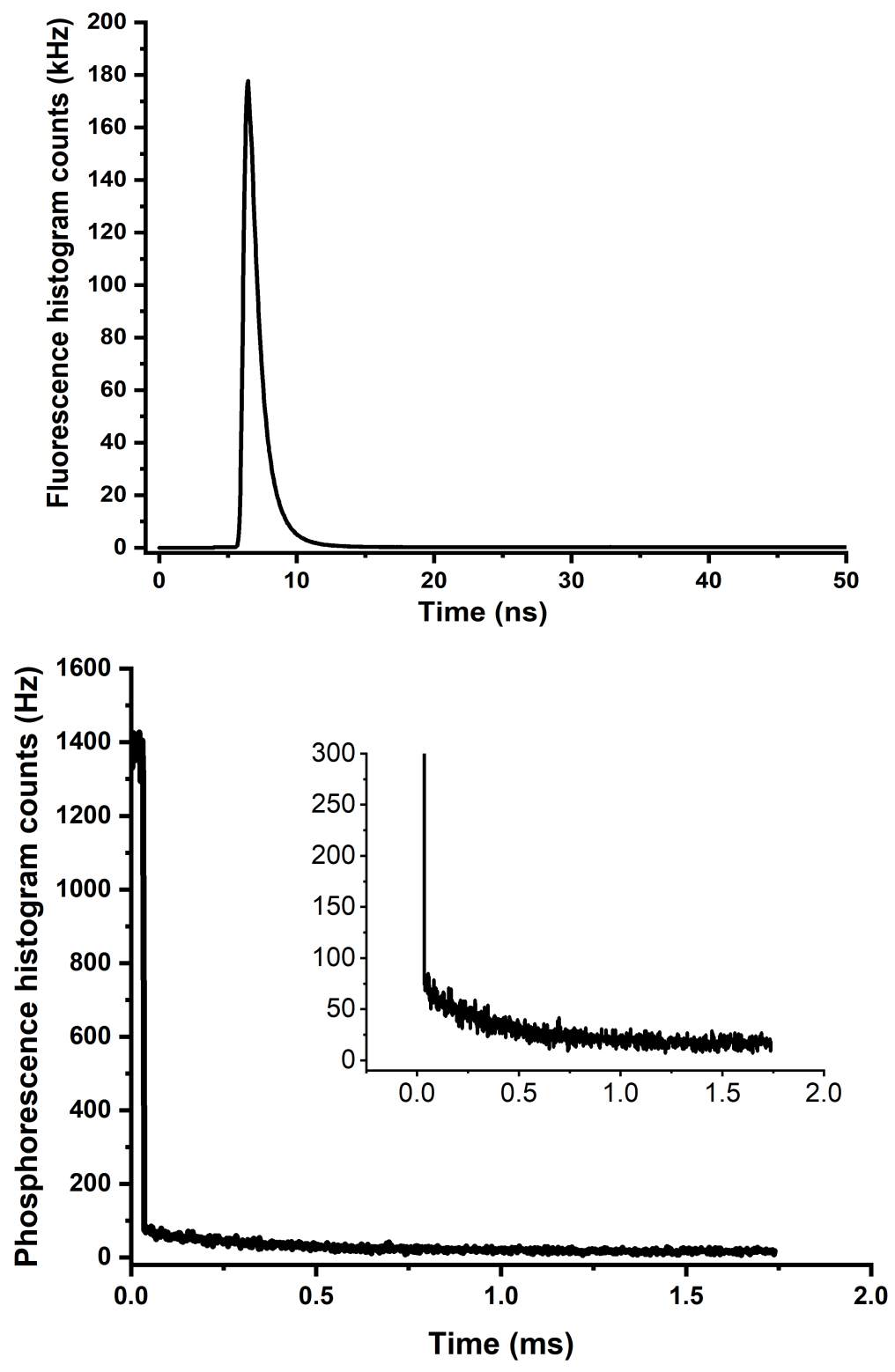

Figure S4: Histograms of simultaneous fluorescence lifetime and phosphorescence lifetime measurements of rose bengal in PVA. The top panel shows the fluorescence decay on a ns timescale and bottom panel shows the phosphorescence on a ms timescale. The inset in the lower panel is simply an expanded y-axis of the lower figure, from 0 to $2000 \mu \mathrm{s}$. 
1. Gratz, H.; Penzkofer, A., Saturable absorption dynamics in the triplet system and triplet excitation induced singlet fluorescence of some organic molecules. Chemical Physics 2001, 263 (2), 471-490.

2. Song, L.; Varma, C. A.; Verhoeven, J. W.; Tanke, H. J., Influence of the triplet excited state on the photobleaching kinetics of fluorescein in microscopy. Biophysical journal 1996, 70 (6), 2959-68.

3. Larkin, J. M.; Donaldson, W. R.; Knox, R. S.; Foster, T. H., Reverse intersystem crossing in rose bengal. II. Fluence dependence of fluorescence following $532 \mathrm{~nm}$ laser excitation.

Photochem Photobiol 2002, 75 (3), 221-8. 